\title{
Experimental Investigation of Static and Dynamic Crosstalk in Trench-Assisted Multi-Core Fibre
}

\author{
Hui Yuan ${ }^{1}$, Arsalan Saljoghei ${ }^{1}$, Tetsuya Hayashi' ${ }^{2}$, Tetsuya Nakanishi ${ }^{2}$, Eric Sillekens ${ }^{1}$, Lidia Galdino ${ }^{1}$, \\ Polina Bayvel ${ }^{1}$, Zhixin Liu ${ }^{1}$, and Georgios Zervas ${ }^{1}$ \\ ${ }^{1}$ Optical Networks Group, UCL (University College London), London WC1E 7JE, United Kingdom \\ ${ }^{2}$ Optical Communications Laboratory, Sumitomo Electric Industries, Ltd., 1, Taya-cho, Sakae-ku, Yokohama 244-8588, Japan \\ h.yuan@ucl.ac.uk
}

\begin{abstract}
We investigate the static and dynamic crosstalk characteristics of TA-MCF in a temperature controlled environment. Results indicate that temperature, PRBS length, modulation format and signaling rate have a significant influence on the properties of crosstalk.

OCIS codes: (060.2300) Fibre measurements, (060.2330) Fibre optics communication; (060.2400) Fibre properties
\end{abstract}

\section{Introduction}

In a move to address the ongoing capacity needs of next generation fibre optic systems, space division multiplexing (SDM) technologies have been developing rapidly in the recent years [1]. Multi-core fibre (MCF) based SDM schemes have shown great benefits in satisfying the capacity, front-panel density, power consumption and cost requirements in data centres [2]. However, the adoption of the MCF is potentially limited by the unwanted inter-core crosstalk (ICXT) between core pairs, which affects the optical signal-to-noise ratio (OSNR) of transmission systems [3].

IC-XT in MCF occurs at stochastically distributed discrete points along the fibre where the phases of the principal and IC-XT signals match [4]. Reference [5] shows that the IC-XT varies over time and the IC-XT variance (VarXT) enforces the system to require higher OSNR margins to prohibit performance degradation or even outages. VarXT can be attributed to the longitudinally varying perturbation along the MCF such as bends, twists and structural fluctuations, which impact the phase matching points (PMPs). Furthermore, it has been shown that Var ${ }_{\mathrm{XT}}$ is strongly affected by the spectral content of the stimulating optical signal and the skew between core pairs [5].

Temperature increases in silica fibre lead to length and refractive index increase by $4.1 \times 10^{-7} \mathrm{mK}^{-1}$ and $1.1 \times 10^{-5} \mathrm{~K}^{-1}$, respectively [6]. These changes alter the skew and dispersion of the link and in turn change the static and dynamic ICXT in MCFs. However, to the best of our knowledge, there has been little study on the impact of temperature variation on IC-XT [7] and the study in [5] was limited by measuring $\operatorname{Var}_{\mathrm{XT}}$ at sub-Hz sampling rates. VarXT was shown to reduce by either increasing the signaling bandwidth in intensity modulated formats or adopting phase modulated formats, where in both cases the power in the residual optical carrier was either reduced or eliminated [8].

In this paper, we present a novel study of the effects of temperature and PRBS length on static and dynamic IC-XT in trench-assisted MCF (TA-MCF). Additionally, we extend the research from [5] on effects of advanced modulation formats and signaling rates on IC-XT. The IC-XT was measured for long periods of time over O-S-C-L bands and results, for the first time, are compared to theoretical calculations, leading to a more comprehensive understanding of the behavior of the IC-XT in MCFs.

\section{Experimental Description}

The cross section of the 8-core TA-MCF employed in this work along with the experimental setup are illustrated in Fig. 1. The fibre, previously described in [9], had a cladding diameter of $180 \mu \mathrm{m}$ and a bending radius of $0.17 \mathrm{~m}$. Our setup consisted of two parts: signal generation (SG) and IC-XT measurement. Two tunable lasers (Fig. 1, SG (a)), one for O-band (1260-1360 nm) with $500 \mathrm{kHz}$ linewidth and one for S-, C-, L-band (1480-1630 nm) with $200 \mathrm{kHz}$ linewidth, were used to generate continuous-wave $(\mathrm{CW})$ laser seed light, respectively. A Mach-Zehnder modulator driven by a pulse pattern generator (PPG) and an electrical sub-system modulated the CW light with OOK and PAM4 signals. The PPG had reconfigurable PRBS pattern with lengths $2^{i}-1(i=7,9,10,11,15,20,23$ and 31$)$ operating at 10 or 25 GBaud. To examine the characteristics of IC-XT with various phase modulated signals, a $100 \mathrm{kHz}$ linewidth external cavity laser (ECL) operating at $1550 \mathrm{~nm}$ was modulated by a dual-polarization I-Q modulator driven by a $92 \mathrm{GS} / \mathrm{s}$ arbitrary waveform generator (AWG) (Fig. 1, SG (b)). The AWG was used to generate various $m$-ary ( $m=4$, 16, 64 and 256) dual-polarization quadrature amplitude modulation (QAM) formats, each capable of operating at signaling rates ranging from 15-80 GBaud. The modulated optical signal was then amplified by an erbium-doped fibre amplifier (EDFA) followed up by a variable optical attenuator (VOA) to control the signal launch power into the MCF fibre. Furthermore, an amplified spontaneous emission (ASE) source followed by a band-pass filter with $1.2 \mathrm{~nm}$ bandwidth (Fig. 1 SG (c)) was used to emulate a broadband signal.

A 48-port optical switch was used to interconnect all input ports of the MCF link via the fan-in device to the various signal sources over single mode single core fibres (SMFs). The switch enabled the generated signals to be sent into any core/cores of the MCF via the fan-in device. The $1 \mathrm{~km}$ TA-MCF was placed in a temperature controlled chamber, which can vary its temperature from $20-80^{\circ} \mathrm{C}$. To measure the IC-XT level, the output of the MCF was fanned-out 
into $8 \mathrm{SMF}$ links. Each link was connected to the input of an 8-port high speed power meter (PM) operating at $40 \mathrm{~Hz}$, capable of detecting power levels as low as $-90 \mathrm{dBm}$. In this setup, the switch and fan-in/out device approximately had $1 \mathrm{~dB}$ and $3 \mathrm{~dB}$ insertion loss, respectively. The optical power of all signals was set to $3 \mathrm{dBm}$ at the input of the optical switch and thus, launch powers through the MCF were not sufficiently high to cause any nonlinearities.

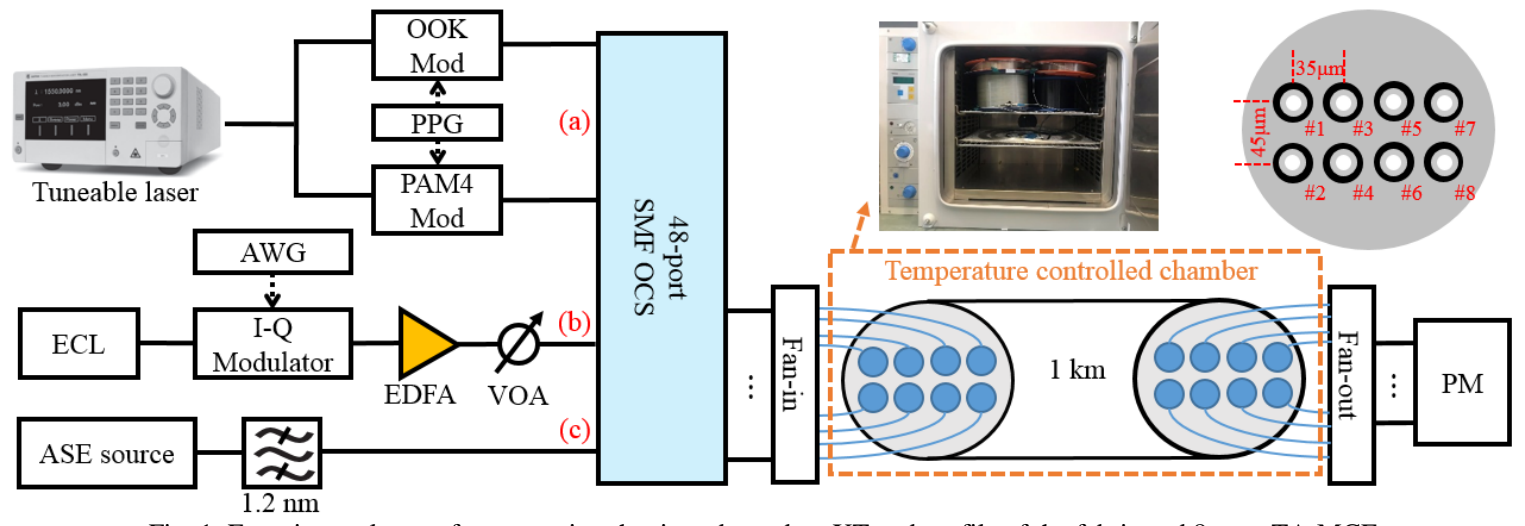

Fig. 1. Experimental setup for measuring the time-dependent XT and profile of the fabricated 8-core TA-MCF

\section{Results and Discussion}

We measured the IC-XT between core 1 (exciting core) and core 3 . Since the core pitch between them was the smallest, the highest (worst case) IC-XT was anticipated. Given that broadband ASE signal leads to lower Var ${ }_{\mathrm{XT}}$ in time [5], a $1550 \mathrm{~nm}$ ASE source was used to explore the effect of temperature on IC-XT. Figure 2(a) depicts the observed accumulated IC-XT in the TA-MCF for temperatures from $20-50{ }^{\circ} \mathrm{C}$ over 1 hour. As it can be seen, a $30^{\circ} \mathrm{C}$ increase in temperature translates into a $1.5 \mathrm{~dB}$ increase in average level of IC-XT and $0.3 \mathrm{~dB}$ reduction in Var ${ }_{\mathrm{XT}}$. Based on the theoretical work carried in [10] for TA-MCFs, it can be deduced that the slightest reduction in the refractive index difference between the core and the cladding can lead to large IC-XT increases between core pairs (e.g. a refractive index variation of $5 \times 10^{-5}$ can lead to $>1 \mathrm{~dB}$ IC-XT increase over $1 \mathrm{~km}$ of fibre). Thus, in our speculation, the average level of IC-XT increase is due to index contrast reduction, and the VarXT reduction due to skew increase [5]. A similar trend is observed in Fig. 2(b), which presents the effects of temperature and operational wavelength on the average IC-XT induced by $25 \mathrm{G}$ OOK signals operating at different wavelengths (each point measured for 1 hour). Figure 2(b) also shows that IC-XT increases by up to $17 \mathrm{~dB}$ over S-, C- and L-bands, which translates into crosstalk-wavelength dependent coefficient of $0.113 \mathrm{~dB} / \mathrm{nm}$. The reason for this dependence of IC-XT on wavelength is that mode field diameter enhances with longer wavelengths, causing further leakage into neighboring cores. Furthermore, it is observed that as the operational wavelength moves from O-band to S-, C- and L-bands, the enhancement of IC-XT as a result of temperature increase also increases by over $100 \%$ (averaging from $0.06 \mathrm{~dB} / \mathrm{K}$ to $0.13 \mathrm{~dB} / \mathrm{K}$ ). This is because the average IC-XT caused by O-band signals after $1 \mathrm{~km}$ of MCF transmission $(\sim-81.5$ to $-67.5 \mathrm{~dB}$, estimated based on [10]) is dominated by the IC-XT induced by the fan-in/out devices ( -58 dB at $1310 \mathrm{~nm}$, measured), which are left outside the chamber (no temperature shift). Moreover, it should be mentioned that, as the sensitivity of the coupling coefficient (parameter for IC-XT calculation) of the utilized MCF to the wavelength change varies in different bands, the slope of the indicator line (black line) for S-, C-, L-band is smaller than that of the O-band.

Figure 2(c) shows the effect of PRBS length on the IC-XT induced by the $25 \mathrm{G}$ OOK signals operating at $1550 \mathrm{~nm}$, with each point giving the average IC-XT value, measured over 1-hour period. It can be seen that an increase in the PRBS length from $2^{7}-1$ to $2^{31}-1$ leads to over $4 \mathrm{~dB}$ of decrease in average IC-XT at $30^{\circ} \mathrm{C}$ and $6 \mathrm{~dB}$ in $50{ }^{\circ} \mathrm{C}$. These results show that the longer the PRBS length, the lower the IC-XT. The reduction of IC-XT with the increase of the PRBS length can be explained in two ways: (i) an increase in the randomness in the transmission pattern, reducing the coherent interferences and, (ii) an increase in the spectral content in the frequency domain as a result of higher spectral sampling. Finally, as shown in Fig. 2(d), the VarXT for different light sources and modulation formats was measured. Each point on this graph is a result of a 12-hour measurement, and all the results were obtained at $1550 \mathrm{~nm}$. According to [7], the lower the bandwidth of optical signals, the higher the VarXT. Thus, as shown in Fig. 2(d), IC-XT measured using a narrow $\mathrm{CW}$ source has the highest level of dynamicity $(22.58 \mathrm{~dB})$, while the broadband ASE light achieves the smallest dynamic range $(0.916 \mathrm{~dB})$, which sets the lower bound. The variability of IC-XT in OOK, PAM and QAM signals is also studied. It is observed that QAM formats have the least impact on Var ${ }_{\text {XT. }}$ OOK and PAM4 signals have strong residual carriers constraining part of the optical power to a finite spectral content, which causes more constructive interference at PMPs and as a result higher stochastic variations in IC-XT intensity. All phase modulated schemes achieve a VarXT level close to that of the ASE case due to the removal of the residual carrier. Among all the QAM formats, QAM4 provides the biggest $\operatorname{Var}_{\mathrm{XT}}$ and QAM256 achieves the least. PAM4 across all studied signalling 
rates exhibits a higher VarXT compared to the OOK signals. This is because more non-zero intensity levels in PAM4 leads to over $30 \%$ increase in the optical carrier-to-signal ratio, which increases the probability of attaining a non-zero level over PMPs across the fibre. The reduction in VarXT, as a result of an increase in the signalling rate by 15 GBaud, is also evident for all intensity modulated signals, leading to reductions in excess of $5 \mathrm{~dB}$ for both OOK and PAM4. Note that Fig. 2 (d) does not include the evolution of $\operatorname{Var}_{\mathrm{XT}}$ for QAM formats over various signalling rates as the obtained values were only measured for 1 hour and not 12 hours. Nevertheless, the 1-hour observation of XT shows that for every QAM format, increasing the signalling rate from 15 to 80 GBaud leads to a reduction of $>1 \mathrm{~dB}$ in $\operatorname{Var}_{\mathrm{XT}}$.

(a)

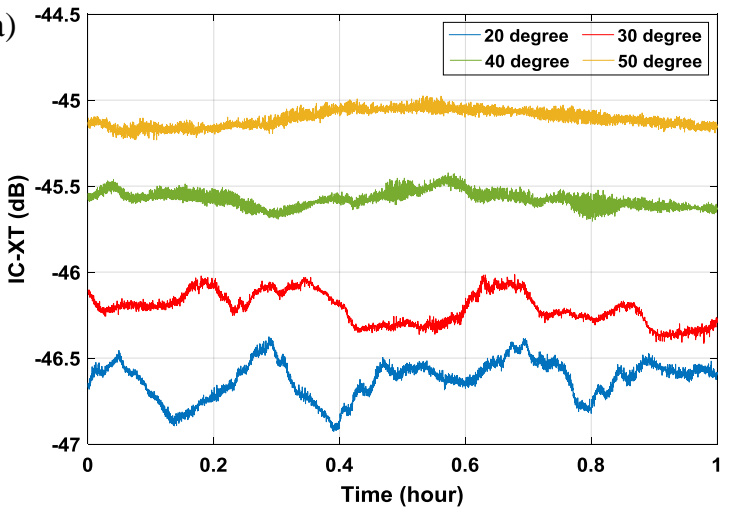

(c)

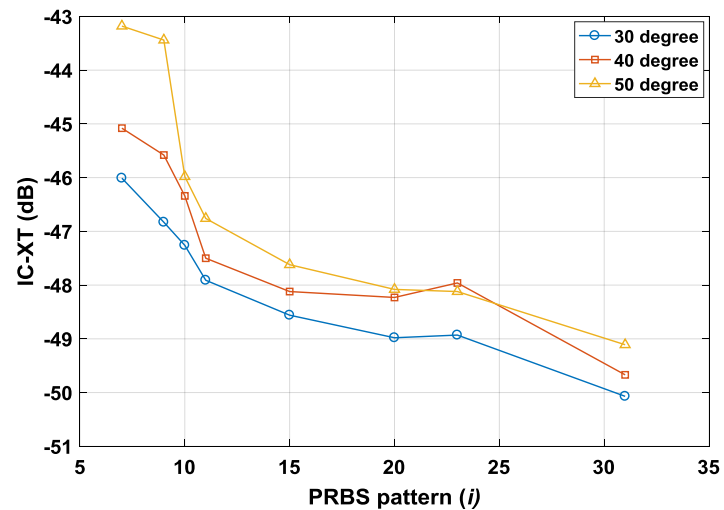

(b)

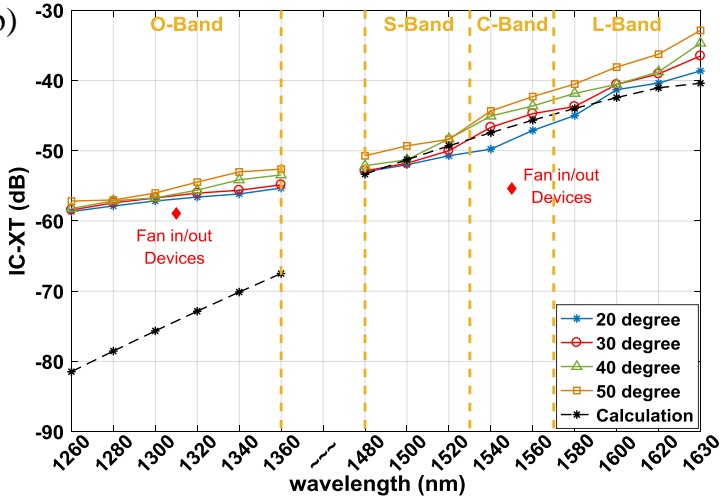

(d)

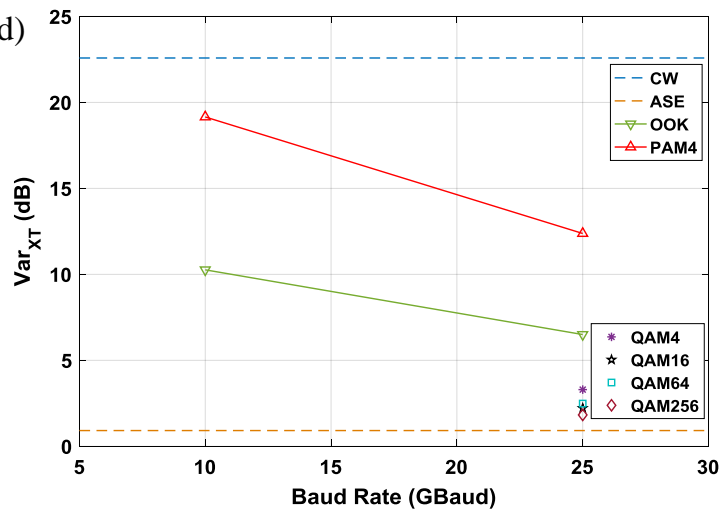

Fig. 2. a) Effect of temperature on the time-dependent IC-XT (ASE), b) Effect of temperature on the wavelength-dependent IC-XT (25G-OOK)

c) Effect of PRBS length on the time-dependent IC-XT (25G-OOK), d) 12-hours IC-XT fluctuations for different modulation formats

\section{Conclusion}

We have experimentally investigated the influence of temperature, PRBS length, operating wavelength, modulation format and signaling rate on static and dynamic IC-XT levels in an 8-core TA-MCF. Results indicate that both static and dynamic IC-XT levels are proportional to the temperature and inversely proportional to the PRBS length, which signifies the importance of temperature and PRBS on IC-XT measurements in laboratory settings. Additionally, experimental results on wavelength-dependent IC-XT accurately fit the theoretical estimation. In terms of IC-XT dynamicity over time, it was found that the higher the temperature or signaling rate, the better the stability. Moreover, the level of IC-XT induced by QAM signals was found to be more stable than that of the OOK and PAM4 signals at signaling rates below 25 GBaud. This is attributed to the reduced carrier power of QAM signals, which leads to a better IC-XT average over the signal band. Furthermore, it was found that IC-XT is over $7 \mathrm{~dB}$ less stable for the PAM4 signaling, which has higher optical carrier-to-signal ratio, compared to the OOK. The understanding and the accurate measurements of IC-XT levels can be used for design of MCF-based data centers or metro networks.

\section{Acknowledgement}

This work is partly supported by EPSRC TRANSNET grant EP/R035342/1, the Royal Academy of Engineering fellowship to Dr L Galdino and EPSRC studentship to E Sillekens. We would also like to thank Santec and Oclaro for the provision of the necessary equipment.

\section{References}

[1] D. J. Richardson, et al., Nature Photon. 7, 354-362 (2013)

[2] H. Yuan, et al., J. Opt. Commun. Netw. 10, 272-288 (2018)

[3] B. J. Puttnam, et al., Photon. J. 8(2), 1-9 (2016)

[4] T. Hayashi, et al., Opt. Express. 19, 16576-16592 (2011)

[5] G. Rademacher, et al., Opt. Express. 25, 12020-12028 (2017)
[6] R. Slavík, et al., Sci. Rep. 5, 15447 (2015).

[7] T. Hayashi, et al., IEICE Trans. Commun. 97, 936-944 (2014)

[8] G. Rademacher, et al., Advanced Photonics, NeTu2B.4 (2017)

[9] T. Hayashi et al. Proc. ECOC. Th.2.A.4 (2017)

[10] F. Ye et al., IEEE Photon. Technol. Lett. 28(1), 27-30 (2016) 